\title{
Formation and light guiding properties of dark solitons in one-dimensional waveguide arrays
}

\author{
Eugene Smirnov, Christian E. Rüter, Milutin Stepić, and Detlef Kip* \\ Institute of Physics and Physical Technology, \\ Clausthal University of Technology, \\ 38678 Clausthal-Zellerfeld, Germany \\ Vladimir Shandarov \\ State University of Control Systems and Radioelectronics, \\ 40 Lenin Ave., 634050 Tomsk, Russia
}

(Dated: October 1, 2018)

\begin{abstract}
We report on the formation of dark discrete solitons in a nonlinear periodic system consisting of evanescently-coupled channel waveguides that are fabricated in defocusing lithium niobate. Localized nonlinear dark modes displaying a phase jump in the center that is located either on-channel (mode A) or in-between channels (mode B) are formed, which is to our knowledge the first experimental observation of mode B. By numerical simulations we find that the saturable nature of the nonlinearity is responsible for the improved stability of mode B. The ability of the induced refractive index structures to guide light of a low-power probe beam is demonstrated.
\end{abstract}

PACS numbers: $42.65 \mathrm{tg}, 42.65 \mathrm{Wi}, 42.82 \mathrm{Et}, 63.20 \mathrm{Pw}$

\footnotetext{
*Electronic address: d.kip@pe.tu-clausthal.de
} 
Nonlinear wave propagation in periodic lattices, which occurs in many different systems in nature [1-4], has attracted great interest in recent years. In these systems the dynamics is dominated by an interplay of diffraction, i.e. tunnelling through adjacent potential wells, and nonlinearity, leading to a large variety of nonlinear effects that have no analogue in bulk media. This increasing interest may be at least partially attributed to recent progress in the investigation of nonlinear wave propagation in optical periodic media, where the ability to engineer optical band structures and diffraction as well as a rather easy experimental observation of nonlinear effects has led to the discovery of many new fundamental features $[5-10]$.

In periodic optical systems including evanescently-coupled waveguide arrays [11, 12], photonic lattices and crystals [13], different types of localized bright structures or lattice solitons have been observed. To name a few, theoretical prediction and experimental realization of spatial gap solitons [14-16], solitons in higher-bands [17], and solitons occupying modes of several bands [18] has been performed. Here we again want to note that the study of these intrinsically localized modes is a universal problem and relevant to many non-optical systems, like localized voltage drops in ladders of Josephson junctions [19], localized modes in antiferromagnetic crystals [20], or localization of matter waves in Bose-Einstein condensates using optically-induced periodic potentials [21].

For bright discrete solitons in Kerr media it has been shown theoretically that, for a given power, two stationary localized modes may exist [23]: a stable mode A centered on a waveguide and an unstable mode $\mathrm{B}$ centered between two neighboring waveguides. On the other hand, we have shown recently that a saturable nonlinearity, for example by using photorefractive nonlinear media, may also support stable propagation of mode B [24, 25]. Very recently, similar predictions have been made for other nonlinear media, for example in cubic-quintic systems [26] and for localized surface waves [27].

As is now well understood, bright lattice solitons can exist either in the region of normal diffraction as a result of a self-focusing (positive) nonlinearity, or in media exhibiting a self-defocusing (negative) nonlinear index change and anomalous diffraction of light in the lattice. Similar to the situation in the bulk $[22,28]$, for dark solitons normal diffraction of a narrow dark notch (a small number of dark elements) on an otherwise homogeneously excited lattice can be balanced by a negative index change, or alternatively anomalous diffraction in a lattice can be compensated by a positive nonlinearity. In periodic systems, the existence 


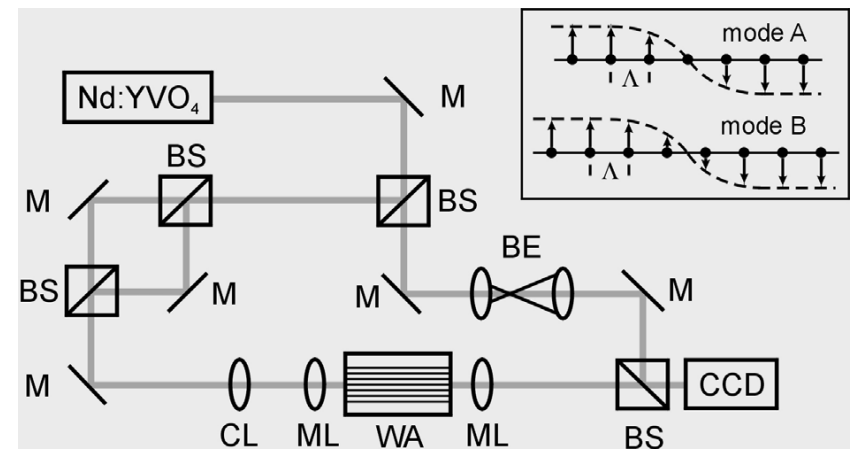

FIG. 1: Experimental setup. M's, mirrors; BS's, beam splitters; CL, cylindrical lens; ML's, microscope lenses; WA, waveguide array; BE, beam expander; CCD, CCD camera. Inset: phase profiles of modes $\mathrm{A}$ and $\mathrm{B}$.

of dark discrete solitons has been investigated theoretically [29], followed only recently by first experimental realizations [16, 30]. As has been recognized already in bulk materials, dark solitons are potential candidates for guiding, steering, and switching of light beams in light-induced refractive index channels. Even more interesting, in discrete media like coupled waveguide arrays, a realization of the above mentioned functions would strongly benefit from the inherent multi-port structure of the array.

In this Letter we investigate formation of dark discrete solitons in one-dimensional waveguide arrays in a material exhibiting a saturable self-defocusing nonlinearity. Localized nonlinear dark modes displaying a phase jump that is located either on (mode A) or in-between (mode B) them are formed, which is - to our knowledge - the first experimental observation of mode B. Numerical simulations that support our experimental findings show that the saturable nature of the used nonlinearity increases stability of mode B when compared to the purely Kerr-type defocusing case. Furthermore, to demonstrate the ability of both types of dark discrete solitons for light steering and switching, guiding of probe beams that are launched into the light-induced refractive index structures is demonstrated.

The waveguide array is fabricated in a $\mathrm{Cu}$-doped lithium niobate crystal, where the saturable defocusing optical nonlinearity arises from the bulk photovoltaic effect. The array that is fabricated by in-diffusion of titanium consists of approximately 250 channels with a width of $4.4 \mu \mathrm{m}$ and a grating period of $8.4 \mu \mathrm{m}[15,31]$.

The experimental setup is sketched in Fig. 1. We use a frequency-doubled $\mathrm{Nd}: \mathrm{YVO}_{4}$ laser with a wavelength $\lambda=532 \mathrm{~nm}$. The light is split into three beams, where two of them are 


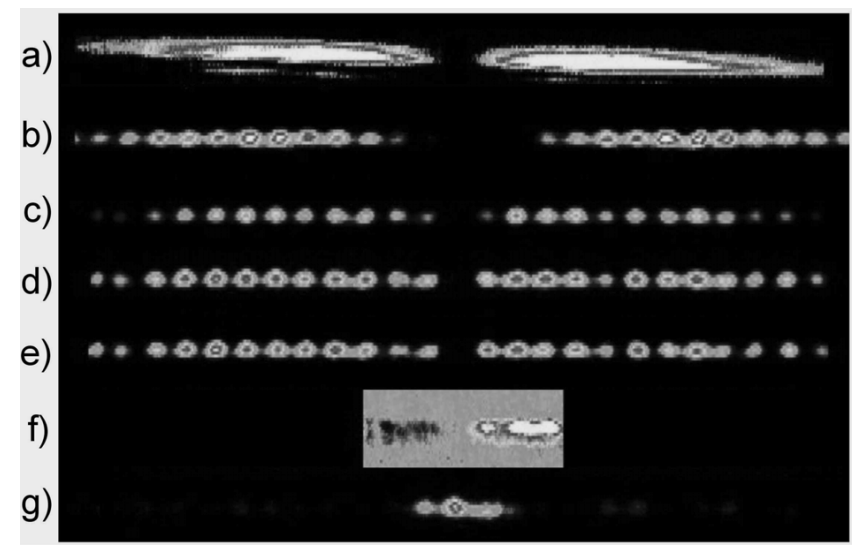

FIG. 2: Discrete diffraction and nonlinear light propagation of mode A: a) intensity distribution on the sample's input face, b) discrete diffraction of mode $\left.\mathrm{A}\left(P_{i n}=1 \mu \mathrm{W}\right), \mathrm{c}\right)$ nonlinear partial focusing at $t=10 \mathrm{sec}$, and $\mathrm{d}$ ) and e) dark soliton formation at $t=20 \mathrm{sec}$ and $t=120 \mathrm{~s}$, respectively $\left.\left(P_{\text {in }}=100 \mu \mathrm{W}\right), \mathrm{f}\right)$ interferogram of the dark soliton shown in part $\left.\mathrm{d}\right)$, and g) guiding of a weak probe beam coupled into the central channel.

formed by a Michelson interferometer and partially superimposed under a small angle on the input face of the waveguide array. In this way a broad beam covering about 25 channels with a small dark notch caused by destructive interference in the overlap region is formed. The center of the formed input beam that experiences a phase jump of $\pi$ in its center can be adjusted either on-channel to excite mode A, or in-between channels to excite mode B (see inset of Fig. 1). A third beam that is expanded to a plane wave with the help of a beam expander is used to investigate the phase structure of the guided light. For this the plane wave interferes with the outcoupled light of the array on the CCD camera.

In a first experiment we investigate the linear and nonlinear propagation of mode $\mathrm{A}$, where the phase discontinuity is located on a lattice element, i.e. the central channel is hardly excited. Here the input width (FWHM) of the dark notch with a total optical power of $100 \mu \mathrm{W}$ is about $\Delta z=25 \mu \mathrm{m}$, covering roughly 4 periods of the lattice and propagating in forward direction with the transverse wave vector component equal to zero. To monitor the input intensity, the photograph in Fig. 2a) shows the reflected light from the sample's input face. In the linear case for low input power, discrete diffraction in Fig. 2b) leads to a broadening of the structure on the homogeneous background, which reaches about 5 channels on the output face after $11 \mathrm{~mm}$ of propagation. The response time of the photovoltaic 
nonlinearity in our sample is about $20 \mathrm{sec}$ for the used input power, thus we are able to monitor the build-up of the discrete dark soliton as a function of time. When the power is increased, first nonlinear de-focusing starts (Fig. 2c) and eventually forms a dark soliton in Fig. 2d) after about $20 \mathrm{sec}$. This structure is stable over times large compared to the build-up time of the nonlinearity (Fig. 2e), taken after $t=120 \mathrm{sec}$ ).

To investigate the nonlinearly induced refractive index structure, we measure the phase profile of the dark soliton by interfering it with a plane wave in Fig. 2f). Obviously, the resulting trapped state has conserved the input phase discontinuity from the input. When the input light is switched off, a probe beam can be coupled into the central input channel. As can be seen in Fig. 2g), the induced structure forms a single mode waveguide guiding the light of this probe beam.

In a second experiment, the waveguide array is laterally shifted by half a lattice period with respect to the interfering input beams, thus exciting mode B with a phase jump inbetween two channels. For a very similar input power as for mode A, an even dark discrete soliton is formed with two dark channels in the center in Fig. 3a). This is to our knowledge the first experimental observation of this intrinsically localized mode. Again, after switching off the pump light the induced structure can be probed by another beam, which in this case covers about 4 channels on the input face. Obviously, this probe beam is guided in two parallel waveguide channels displayed in Fig. 3b). When illuminating the sample with intense white light, the induced refractive index structure is erased and finally the probe beam propagates with normal diffraction in the (undisturbed) lattice in Fig. 3c).

To compare our experimental results with theory, we simulate the light propagation in the array using a nonlinear beam propagation method. For mode A with an input width (FWHM) of $\Delta z=25 \mu \mathrm{m}$, in Fig. 4a) the case of linear diffraction for low input power is

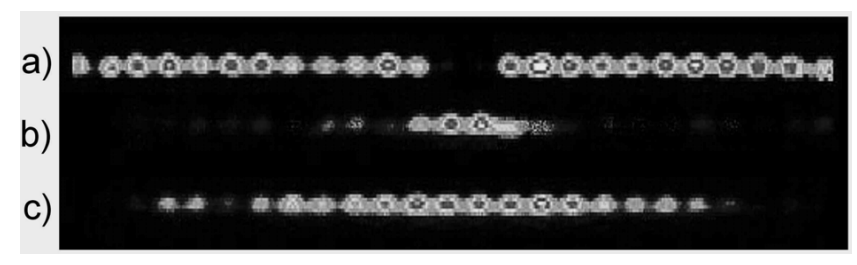

FIG. 3: Nonlinear propagation of mode B: a) dark soliton formation at $t=60 \mathrm{sec}, \mathrm{b})$ guiding of a probe beam, and c) diffraction of the input probe beam in the linear lattice. 


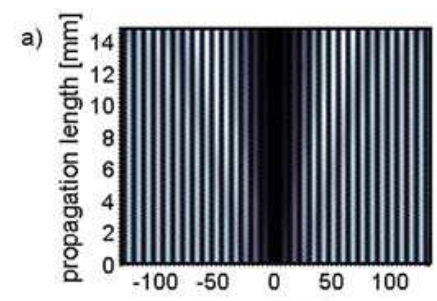

b)
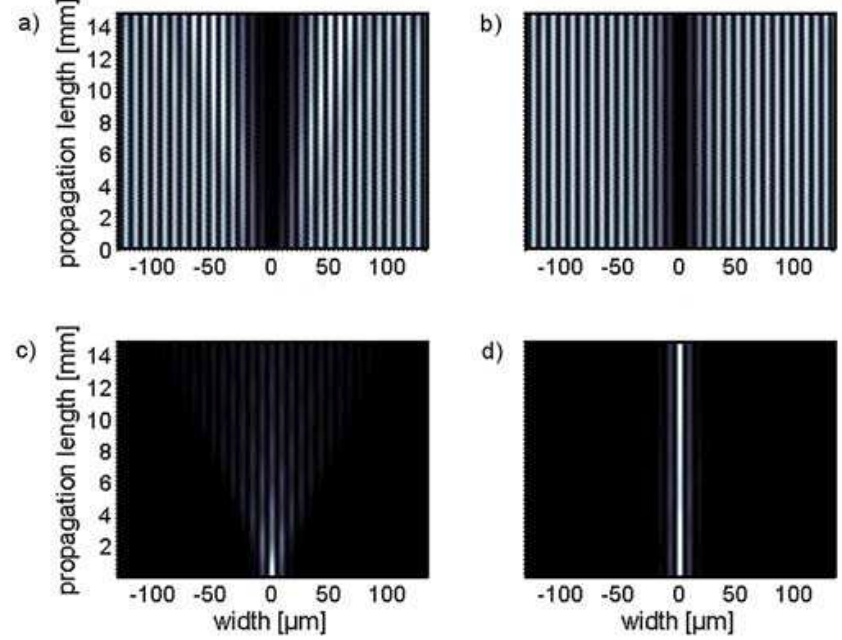

FIG. 4: Simulation of mode A: a) linear diffraction of a dark notch, b) discrete dark soliton formation, c) diffraction and d) guiding of a probe beam, respectively.
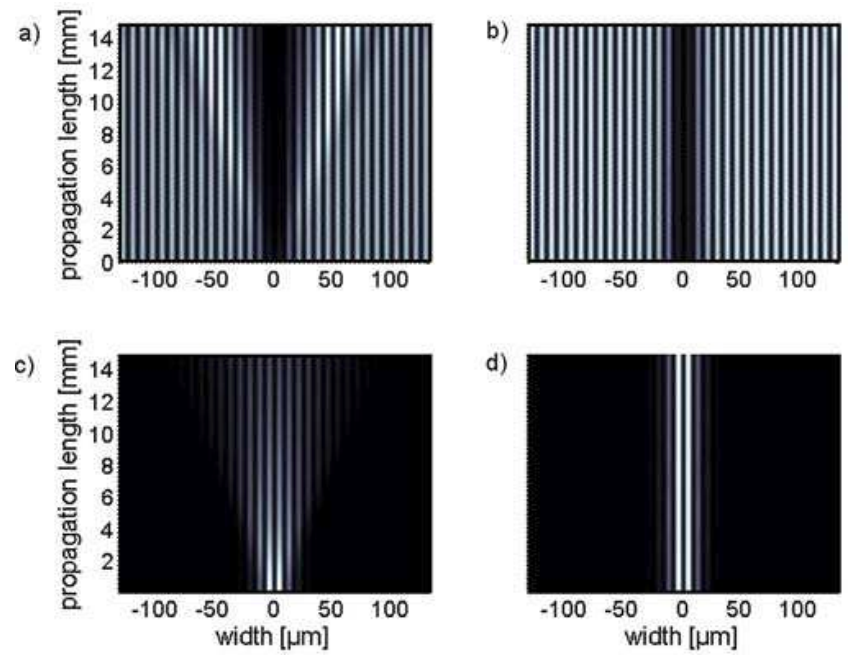

FIG. 5: Simulation of mode B: a) discrete diffraction of a linear beam, b) discrete dark soliton formation, c) diffraction and d) guiding of a probe beam, respectively.

shown. A localized dark soliton is obtained in Fig. 4b) in the nonlinear regime applying a saturable nonlinearity of the form $\Delta n=\Delta n_{0} r /(1+r)$, where $\Delta n_{0}=-1.5 \times 10^{-4}$ is the amplitude of nonlinear index, $r=I / I_{d}=8$ is light intensity ratio with intensity $I$ and dark irradiance $I_{d}$. When the dark soliton is formed, a low-power probe beam that is coupled into the central channel can be guided in the written refractive index distribution of the lattice (Fig. 4d). In the linear case this beam diffracts without guiding (Fig. 4c). 
For mode B and the same nonlinearity as above, linear diffraction and soliton formation are shown in Fig. 5a) and b), in good agreement with the experimental results. For a broader (linear) input probe beam covering about four input channels, the resulting even refractive index distribution shows guiding in the two channels (Fig. 5d)), whereas this beam diffracts to a broad output beam in the linear case.

As has been shown theoretically for the Kerr case [29], mode B experiences instability during propagation. Here instability (i.e., conversion of mode B into mode A) may originate from a small mismatch of the symmetric input condition, for example by a lateral shift of the input light distribution, a small tilt angle of the input beam or a small asymmetry in intensity. However, our simulation results show that the stability of mode B is significantly improved by the saturable form of the nonlinearity, as it has been predicted recently in Ref. [24] for bright discrete solitons.

Two numerical examples of the improved stability are given in Fig. 6. In the upper part (Fig. 6a) and b)) we have added a small tilt angle $\delta \alpha=0.5^{\circ}$ of the input phase front, with all other parameters being the same as in Fig. 5. As can be seen, for a saturable nonlinearity (a) almost stable propagation of mode B is obtained, while for a purely Kerr-type nonlinearity the input mode experiences instability and is converted into mode A.

A similar behavior is observed if we introduce a small asymmetry in the input light

a)

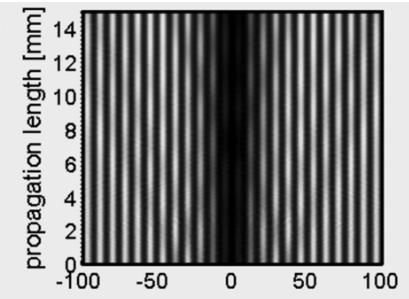

C)

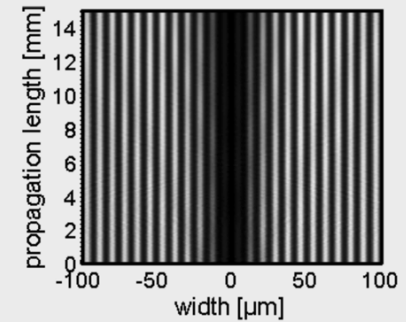

b)

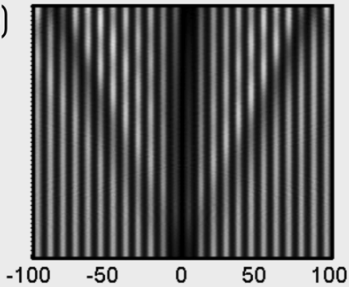

d)

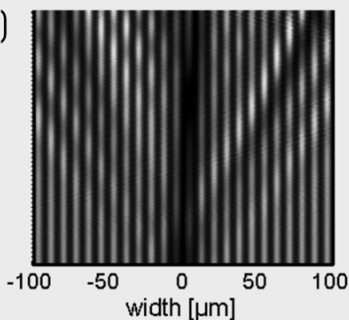

FIG. 6: Comparison of stability of mode B for saturable and Kerr-type nonlinearity. Tilt angle $\delta \alpha=0.5^{\circ}$ of the input phase front for a) saturable and b) Kerr case, and asymmetry $P_{i n, r} / P_{i n, l}=$ 1.03 of power of right and left half of the input beam for c) saturable and d) Kerr case. 
intensity. In Fig. 6c) and d) the right hand side of the input intensity is increased by $3 \%$ relative to that on the left hand side. Obviously, this asymmetry leads to destabilization of mode B in the Kerr case in d), while the same input intensity propagates stable in a saturable nonlinear waveguide array in part c).

To summarize, we have experimentally investigated dark soliton formation in a nonlinear waveguide array with defocusing saturable nonlinearity. Stable nonlinear light propagation of localized dark beams centered either on-site or in-between two channels has been observed experimentally, and our findings are well supported by numerical simulations. It has been shown that the saturable character of the nonlinearity leads to stabilization of mode B. The ability of the induced refractive index patterns to guide light in form of two different stable structures has been demonstrated, which is of great practical interest for the realization of all-optical devices like routers and switches.

\section{Acknowledgments}

We gratefully acknowledge financial support from the German Federal Ministry of Education and Research (BMBF grant DIP-E6.1) and the Deutsche Forschungsgemeinschaft (DFG grants KI482/8-1 and 436RUS17/26/06). D. Kip's e-mail address is: d.kip@pe.tuclausthal.de.

[1] A. J. Sievers and S. Takeno, Phys. Rev. Lett. 61, 970 (1988).

[2] A. Xie, L. van der Meer, W. Hoff, and R. H. Austin, Phys. Rev. Lett. 84, 5435 (2000).

[3] D. N. Christodoulides, F. Lederer, and Y. Silberberg, Nature 424, 817 (2003).

[4] D. K. Campbell, S. Flach, and Yu. S. Kivshar, Physics Today 57, 43 (2004).

[5] H. S. Eisenberg, Y. Silberberg, R. Morandotti, and J. S. Aitchison, Phys. Rev. Lett. 85, 1863 (2000).

[6] D. N. Christodoulides and R. I. Joseph, Opt. Lett. 19, 794 (1988).

[7] H. S. Eisenberg, Y. Silberberg, Y. Morandotti, R. Boyd, and J. S. Aitchison, Phys. Rev. Lett. 81, 3383 (1998).

[8] J. W. Fleischer, G. Bartal, O. Cohen, T. Schwartz, O. Manela, B. Freedman, M. Segev, H. 
Buljan, and N. K. Efremidis, Opt. Express 13, 1780 (2005).

[9] M. Stepić, C. Wirth, C. E. Rüter, and D. Kip, Opt. Lett. 31, 247 (2006).

[10] O. Cohen, G. Bartal, H. Buljan, T. Carmon, J. W. Fleischer, M. Segev, and D. N. Christodoulides, Nature 433, 500 (2005).

[11] J. W. Fleischer, T. Carmon, M. Segev, N. K. Efremidis, and D. N. Christodoulides, Phys. Rev. Lett. 90, 023902 (2003).

[12] F. Chen, M. Stepić, C. E. Rüter, D. Runde, D. Kip, V. Shandarov, O. Manela, and M. Segev, Opt. Exp. 13, 4314 (2005).

[13] S. F. Mingaleev and Yu. S. Kivshar, Phys. Rev. Lett. 86, 5474 (2001).

[14] D. L. Mills and S. E. Trullinger, Phys. Rev. B 36, 947 (1987).

[15] Yu. S. Kivshar, Opt. Lett. 20, 1147 (1993).

[16] D. Mandelik, R. Morandotti, J. S. Aitchison, and Y. Silberberg, Phys. Rev. Lett. 92, 093904 (2004).

[17] O. Manela, O. Cohen, G. Bartal, J. W. Fleischer, and M. Segev, Opt. Lett. 29, 2049 (2004).

[18] O. Cohen, T. Schwartz, J. W. Fleischer, M. Segev, D. N. Christodoulides, Phys. Rev. Lett. 91, $113901(2003)$

[19] E. Trias, J. J. Mazo, and T. P. Orlando, Phys. Rev. Lett. 84, 741 (2000).

[20] U. T. Schwarz, L. Q. English, and A. J. Sievers, Phys. Rev. Lett. 83, 223 (1999).

[21] A. Trombettoni and A. Smerzi, Phys. Rev. Lett. 86, 2353 (2001).

[22] G. A. Swartzlander, D. R. Andersen, J. J. Regan, H. Yin, and A. E. Kaplan, Phys. Rev. Lett. 66, 1583 (1991).

[23] Yu. S. Kivshar and D. K. Campbell, Phys. Rev. E 48, 3077 (1993).

[24] Lj. Hadžievski, A. Maluckov, M. Stepić, and D. Kip, Phys. Rev. Lett. 93, 33901 (2004).

[25] A. Maluckov, M. Stepić, D. Kip, and Lj. Hadžievski, Eur. Phys. J. B45 , 539 (2005).

[26] R. Carretero-González, J. D. Talley, C. Chong, and B. A. Malomed, Physica D - Nonlinear Phenomena 216, 77 (2006).

[27] Y. V. Kartashov, V. A. Vysloukh, and L. Torner, Phys. Rev Lett. 96, 073901 (2006).

[28] Yu. S. Kivshar, IEEE J. Quant. Electron. 29, 250 (1993).

[29] Yu. S. Kivshar, W. Królikowski, and O. A. Chubykalo, Phys. Rev. E 50, 5020 (1994).

[30] R. Morandotti, H. S. Eisenberg, Y. Silberberg, M. Sorel, and J. S. Aitchison, Phys. Rev. Lett. 86, 3296 (2001). 
[31] C. E. Rüter, J. Wisniewski, and D. Kip, submitted to Optics Letters (2006). 